\title{
Evaluation Method of Emulsion Using Chemical Oxygen Demand
}

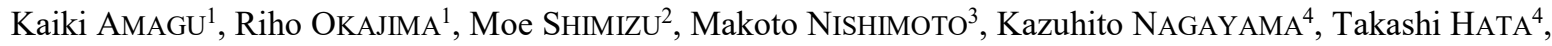 \\ Yusuke NisHIUCHI ${ }^{4}$, and Kaori TADA ${ }^{4 *}$ \\ ${ }^{1}$ Advanced Course of Material Engineering, National Institute of Technology (KOSEN), Kochi College, 200-1 Monobe Otsu, \\ Nankoku-city, Kochi 783-8508, Japan \\ ${ }^{2}$ Department of Material Science, National Institute of Technology (KOSEN), Wakayama College, 77 Noshima, Nada-cho, \\ Gobo-city, Wakayama 644-0023, Japan \\ ${ }^{3}$ Department of Applied Chemistry and Biochemistry, National Institute of Technology (KOSEN), Wakayama College, 77 Noshima, \\ Nada-cho, Gobo-city, Wakayama 644-0023, Japan \\ ${ }^{4}$ Department of Social Design Engineering, National Institute of Technology (KOSEN), Kochi College, 200-1 Monobe Otsu, \\ Nankoku-city, Kochi 783-8508, Japan
}

\begin{abstract}
The "gas-liquid two-phases mixed flow" mechanism that fine bubble generation uses rapidly swirling water, which shear gas and making small bubbles in water. Based on this mechanism, sending liquid such as oil into the swirling water instead of gas and it able to be emulsified. At present, evaluation methods for emulsions are using particle size distribution and dispersion stability. However, the emulsion with bad separation behavior has a problem to catch only some dispersed oil particles and use them as a whole evaluation. In this study we examined emulsion evaluated by chemical oxygen demand (COD) to express numerical evaluation including the oil particles that cannot be dispersed. Using three emulsions of only one oil, an anionic and a nonionic emulsifier were mixed and added to the oil, an anionic and another kind of the nonionic emulsifier were mixed and added to the oil. It was confirmed that COD decreased and type of only one oil was the most evaporate. These results lead to the conclusion that the performance of the emulsion could be expressed numerically, including the oil particles which could not be dispersed. We also directly analyzed evaporate components and compared them with COD results.
\end{abstract}

\section{Introduction}

An emulsion is a mixture of two or more liquids that are normally immiscible such as oil and water. The emulsion which oil dispersed in water called $\mathrm{O} / \mathrm{W}$ type emulsion. On the other hand, the emulsion which water dispersed in oil called W/O type (Kitahara and Furusawa, 1979). Example of emulsions in the field of food are milk and mayonnaise. Emulsion is used in various fields, such as cosmetics, medical products.

Recently, it was confirmed that fine bubbles have a diameter under $100 \mu \mathrm{m}$ have properties different from those of $\mathrm{mm}$ or $\mathrm{cm}$ sized bubbles. The "gas-liquid twophases mixed flow" mechanism that fine bubble generation uses rapidly swirling water that shear gas and making small bubbles in water. Based on this mechanism, sending liquid such as oil into the swirling water instead of gas and it able to be emulsified. This time, the emulsion was made by this mechanism.

Main evaluation methods for emulsions are using particle size distribution and dispersion stability.
However, the emulsion with bad separation behavior has a problem to catch only some dispersed oil particles and use them as a whole evaluation. This can be visualization. Nevertheless it is difficult to express numerical data. In this study we investigated emulsion evaluated by chemical oxygen demand (COD) to express numerical evaluation including the oil particles which cannot be dispersed. We also directly analyzed evaporate components and compared them with COD results.

\section{Experimental Method}

\subsection{Producing of emulsion using liquid-liquid two-phases mixed flow system}

In this study we examine emulsion were made by the liquid-liquid two-phases mixed flow system applying the gas-liquid two-phase mixed flow system, which is the mechanism of fine bubble generation. Figure 1 shows the device outline. In the gas-liquid two-phases mixed flow system, the gas is refined by the shear stress

* Corresponding author: tada@ge.kochi-ct.ac.jp 
of rapidly swirling liquid. It is possible to send liquid instead of gas. The dispersed phase is refined by the shear stress of the continuous phase and the dispersed phase. And $\mathrm{O} / \mathrm{W}$ emulsion which oil is dispersed in water could be made.

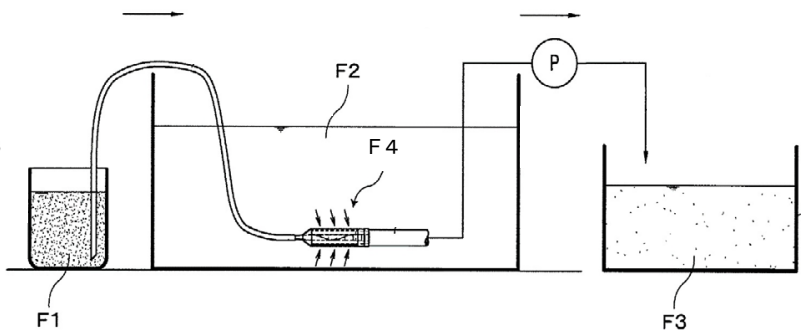

Figure 1. Liquid to liquid two phases mixed flow system (F1: disperse phase (oil), F2: continuous phase (water), F3: formed emulsion, F4: generation nozzle)

\subsection{Producing of emulsion using conventional machine}

We made a comparison between this technology and conventional machine. The following shows the conventional machine.

We used homogenizer, rotor stator type mixer and ultrasonic emulsifying machine.

First, homogenizers emulsify oil and water by high pressure. At that time, liquid is injected from the valve gap. This time, the pressure applied to the valve was 300 bar to produce emulsion.

Second, rotor stator type mixers stir and make emulsion. A rotor rotating at high speed with a conical mixer cuts the liquid by stirring it. The rotor speed was $300 \mathrm{rpm}$ for 5 minutes.

Third, ultrasonic emulsifying machines apply ultrasonic waves to oil and water. The relatively low frequency ultrasonic emulsification of several tens of $\mathrm{kHz}$ vibrates the unstable contact interface such as water and oil, and formed droplets. They are divided by the generated cavitation. On the other hand, high frequency ultrasonic waves of $\mathrm{MHz}$ or more, the size of the generated cavitation is in the submicron range. Therefore it is considered to be effective for further refinement of the emulsion droplets that have already been formed (Atobe, 2012). Based on this, it was pre-emulsification for 5 minutes with low-frequency ultrasonic wave (45 $\mathrm{kHz}$ ). After that similarly performed for 5 minutes with high frequency $(1.6 \mathrm{MHz})$.

\subsection{Evaluation of emulsion}

We evaluated made emulsion. The following shows the evaluation method.

Evaluation methods of emulsion are particle size distribution, transmittance and dispersion stability index and chemical oxygen demand (COD).

First, particle size distribution shows the size and number of particles. The measuring device used SALD 7100 nano (Shimadzu Corporation). This device uses a laser diffraction and scattering equation. They measure the particle size by the scattered light when light strikes the particle.

Second, transmittance shows transparency. The measuring device used an ultraviolet-visible spectrophotometer V-630 (JASCO Corporation). The measurement wavelength was $660 \mathrm{~nm} .0 .5 \mathrm{~mL}$ sample take to the cell and add $3 \mathrm{ml}$ of ion-exchanged water.

Third, dispersion stability index is an indicator of the stability of the emulsion. In the case of $\mathrm{O} / \mathrm{W}$ type emulsion, the oil droplets accelerate to float and coalescence over time. Since oil density is bigger than water density. This phenomenon is called creaming behavior, and its speed is a measure of dispersion stability (Tasaki, 2014). In order to observe the creaming behavior, the emulsion was subjected to centrifugal separation. The instantaneous change in transmitted light quantity was regarded as the positional change of the particle group. Thus, the dispersion stability index was calculated. Device uses LUMi Fuge (L.M.F).

Fourth, COD indicates the amount of organic matter in the target.

\subsection{Producing of Emulsion}

The dispersed phase and the continuous phase are stirred or mixed as they are, one liquid is refined to make emulsion, but when it is left, the droplets coalesce immediately and separate into the two liquids as before.

The third substance added to stabilize the emulsion is called emulsifying. It also reduces the energy required to refine the liquid and facilitates emulsification.

As a concrete experimental operation, the dispersed phases used was of three types: sample (1) only one oil, sample (2) an anionic and a nonionic emulsifier were mixed and added to the oil, sample (3) an anionic and another kind of the nonionic emulsifier were mixed and added to the oil. The continuous phase was ionexchanged water, and the above three dispersed phases were emulsified at 1 vol.\%. Also, the oil used was volatile. However, rotor stator type mixers are structural problems and cannot make oil only. Samples were measured every three days for nine days and stored at 60 ${ }^{\circ} \mathrm{C}$.

\section{Results and discussion}

\subsection{Temporal change of dispersed phase by COD}

As a result of particle size distribution for nine days, the emulsion in sample (1) was polydispersed. On the other hand, the emulsions in samples (2) and (3) were monodispersion and a particle size is about $50 \mathrm{~nm}$. In other words, the particle size of the emulsion in sample (1) is more than larger the emulsion in sample (2) or (3). And stability is also same as particle size.

Passed time was taken on the horizontal axis, and the transmittance (Figure 2), particle size distribution (Figure 3), and the dispersion stability index (Figure 4) are plotted on the vertical axis. It can be confirmed that the transmittance (Figure 2) and dispersion stability 
(Figure 4) are higher with time. As to particle size distribution (Figure 3), it was found that the mode diameter of the emulsions in sample (2) and (3) remained stable without change. However the mode diameter of the emulsion in sample (1) was not stable. Thus, the emulsion tends to be stabilized with time.

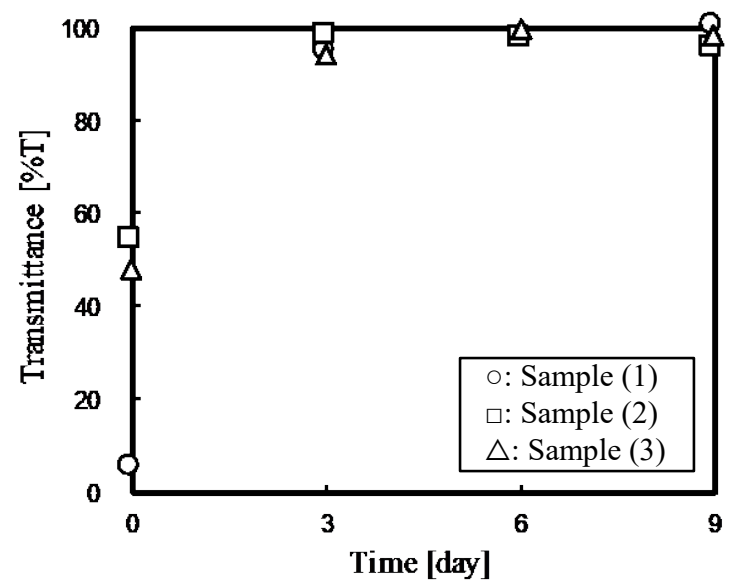

Figure 2. Transmittance (this technology)

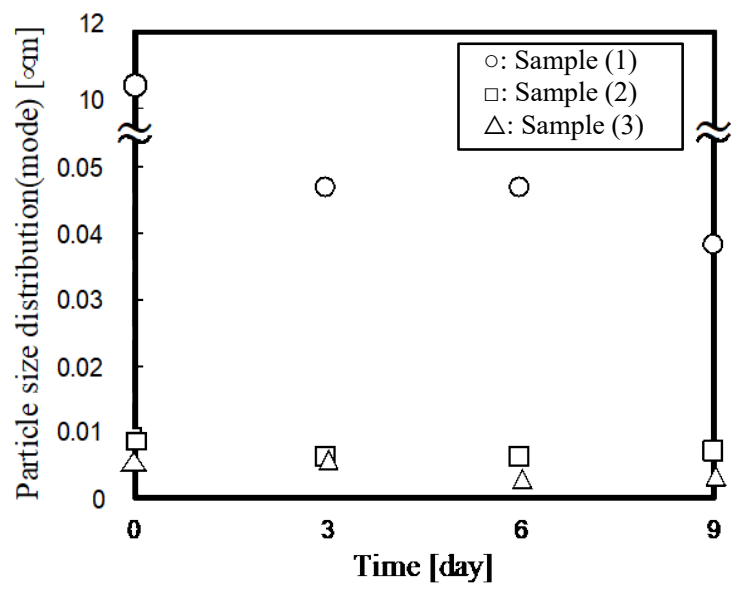

Figure 3. Particle size distribution (mode, this technology)

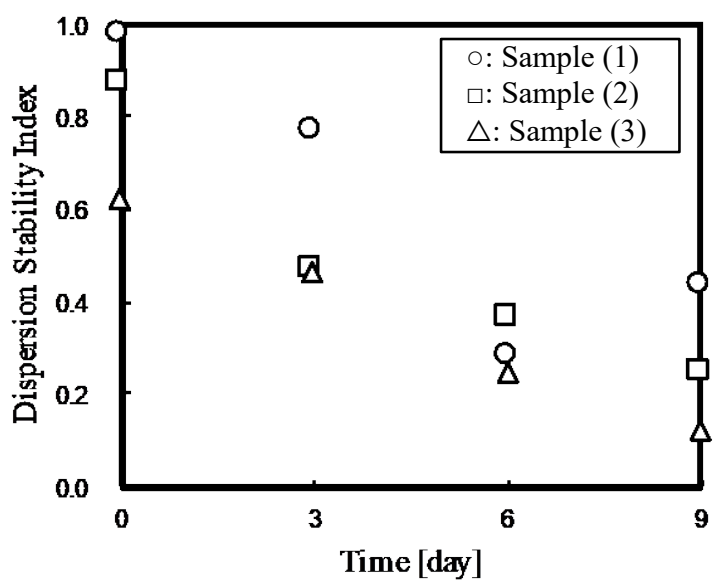

Figure 4. Dispersion stability index (this technology)
Next, it was confirmed that the change in oil amount calculated from COD over time (Figure 5). As to the emulsion in sample (1), the oil was completely vaporized. And the emulsions in sample (2) and (3) were evaporated about $1000 \mathrm{mg} \mathrm{L}^{-1}$.

In Figure 3, because an emulsion of about $10 \mu \mathrm{m}$ is observed in sample (1), it is considered that remarkable creaming and vaporized from the upper layer occurred due to the relatively large particle size. It is supported by the results of increased transmittance and decreased COD value according to a decrease in emulsion concentration.

From this result, it was found that amount of the oil dispersed phase was calculated from COD, and the behavior of the dispersed phase could be confirmed. Emulsion performance is relative to COD results.

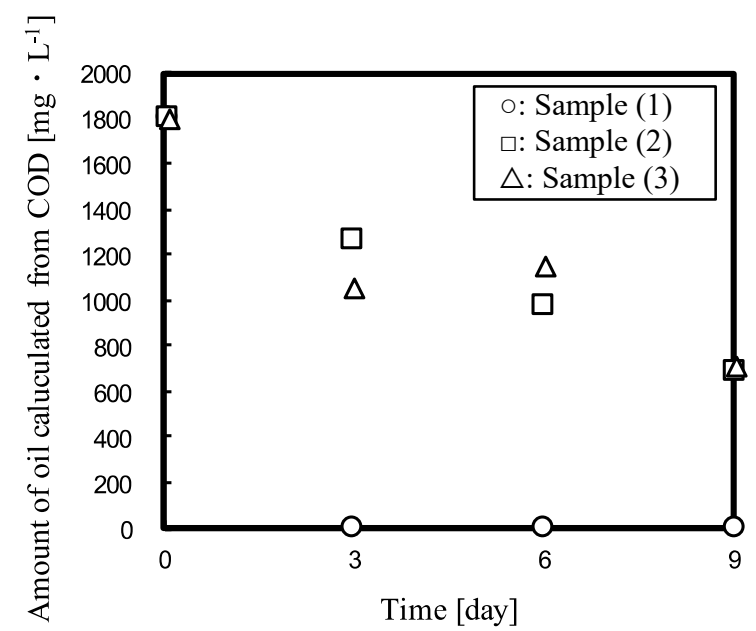

Figure 5. The amount of oil calculated from COD (this technology)

\subsection{Emulsion tend of this technology and conventional machines}

Similar to this technology, it was confirmed that the particle diameter was smaller and the transmittance and dispersion stability were higher on conventional machines for 9 days. For example, the emulsion made by homogenizer, we show the particle size distribution (Figure 6) and a result of COD (Figure 7).

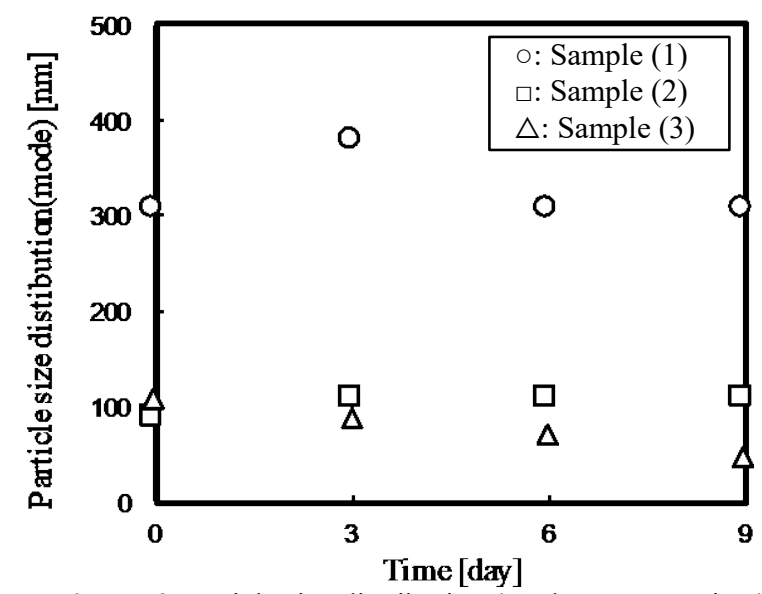

Figure 6. Particle size distribution (mode, Homogenizer) 


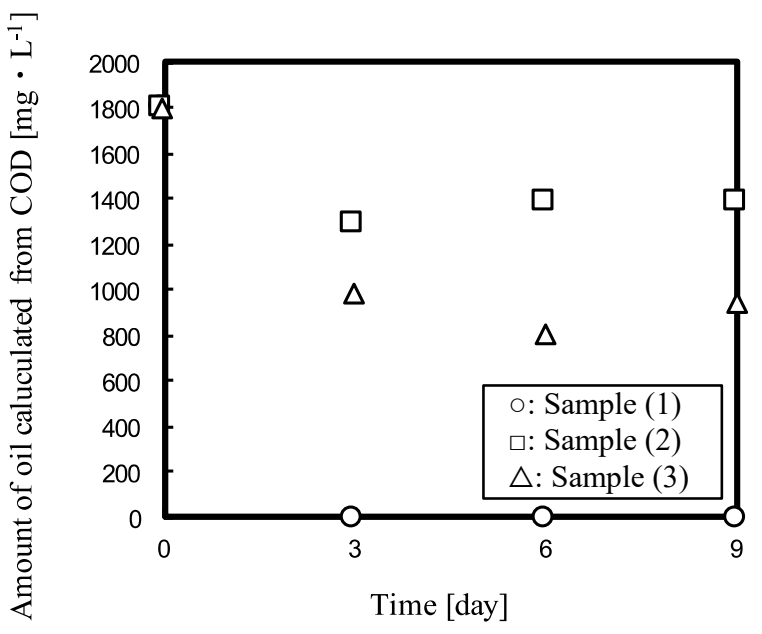

Figure 7. The amount of oil calculated from COD (Homogenizer)

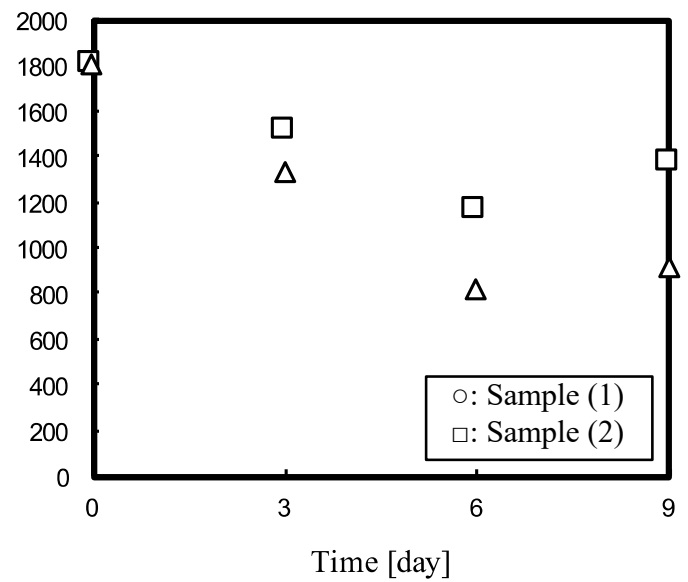

Figure 8. The amount of oil calculated from COD (Rotor stator type mixer)

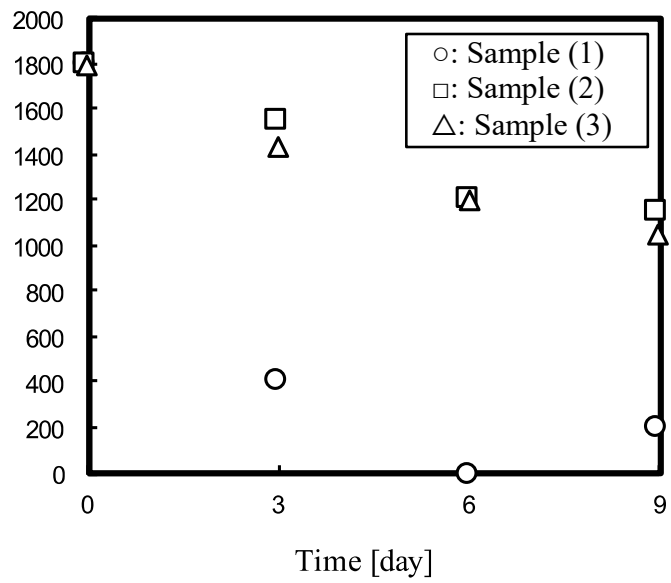

Figure 9. The amount of oil calculated from COD (Ultrasonic emulsifying machines)

In addition, a result of COD of other machines show Figure 8 and Figure 9.

As a result, the emulsion performance by conventional machines is same as the emulsion performance by liquid-liquid two-phases mixed flow system over time.

Measurements other than COD consider that the slight remaining fine particle were captured and reflected in the results on the emulsion in sample (1). It appears stability, over time. Therefore we should not overlook that decreasing oil. The emulsion such as sample (1) is that large oil particles are evaporated rapidly and only small fine particles remain. In comparison with the emulsion such as sample (1), the emulsion such as sample (2) or (3) is that oil evaporates slowly because the particles are small.

\section{Conclusion}

In this study, we measured and evaluated particle size distribution, transmittance and dispersion stability of $\mathrm{O} / \mathrm{W}$ type emulsions made by liquid-liquid two-phases mixed flow system. And temporal changes of emulsions were evaluated by COD. As time passed, the particle size was smaller. Besides, the transmittance and dispersion stability are higher. In other words, the emulsions tend to be stability from these results. However the amount of oil calculated from COD decreased, the emulsion became thinner due to creaming behavior and evaporated oil. Dispersion oil and amount of evaporated oil are associated with oil type and the combination of emulsifiers.

In addition, we evaluated the emulsion which made by conventional machines with same composition as this technology. As a result, it was found that the particle size was smaller and the transmittance and the dispersion stability were higher with time, but the oil dispersion amount was lower than COD, even the conventional machine, same as this technology. The emulations by conventional machines are also about the same performance.

From these results, the evaluation method using COD can conform the concentration of the dispersed phase remaining in solution. The evaluation of COD values is effective in systems without impurities. COD is important when evaluating the emulsion.

\section{Acknowledgments}

This work was supported by Kochi Prefecture industryacademia-government collaboration commercialization support project.

\section{References}

Kitahara, A. and K. Furusawa; Chemistry of Dispersion and Emulsification Systems, p. 54, Engineering Books Co Ltd., Tokyo, Japan (1979)

Atobe, M.; "Preparation of Surfactant-free Nanoemulsion Using Tandem Acoustic Emulsification and Its Application", Oleoscience, 12(6), 233-239 (2012)

Tasaki, H.; Characterization of Emulsion and Development of New Product, Utilization for Quality Control, pp. 128, 139, 152, Technical Information Institute Co. Ltd., Tokyo, Japan (2014) 